\title{
Dynamics Analysis and Trajectory Planning of Erecting mechanism
}

\author{
YuXi Liu", a , Qinhe Gao ${ }^{1, b}$ and Xiangrui Chen ${ }^{1, c}$ \\ ${ }^{1}$ Xi'an Research Institute of High-Tech, Xi'an 710025, China \\ 849852677@qq.com,821346002@qq.com,527568786@qq.com.
}

Keywords: Leveraged Balance on Erecting mechanism. Dynamics analysis. ADAMS Modeling. Trajectory Planning.

\begin{abstract}
The single-stage cylinder leveraged balance erecting solutions was adopted to solve multistage cylinder change-stage impact on the erecting mechanism which mathematical and simulation model is build. The dynamics performance is very significant for stability and rapidity of Erecting mechanism, therefore, dynamics analysis for the erecting mechanism is made in a condition of setting its movement rules of trajectory planning. In the end, a better erecting trajectory is elected due to dynamics simulation which is compared by using ADAMS software for five movement rules. This would be a important reference to improving performance of the erecting mechanism on the fast and smooth.
\end{abstract}

\section{Introduction}

The development of modern military technology ${ }^{[1]}$ shows increasing demand for rapid response capability of military equipment, and it depends largely on the rapid erection capability for mobile missile weapons to reduce the reaction time and achieve the quick launching after arriving at the launching site ${ }^{[2]}$, so it shows great request for the rapidly and stability of the motion of the missile erecting mechanism.

Currently, uniform angular velocity motion is mainly method in the driving motion of the erecting mechanism, which have a greater impact on erecting process so that it can affect the rapid and damage the structure ${ }^{[3][4]}$. In order to improve the rapidty and stability of the erecting mechanism, the trajectory planning that figure out the motion movement of the electric cylinder is greatly necessary ${ }^{[5][6]}$. W.H Tang set up the dynamic equation on dynamics model and have an analysis on inverse dynamics by planning the relationship between the erecting angle and the running time. Aimed at the problem of long time of erection, G. Tian studied on trajectory planning with the goal of the shortest time on missile erector, achieving the rapidity of erecting process. X.Y Wu ${ }^{[7]}$ planned the Electric cylinder extending speed from the point of view on power and torque of the motor, reducing the time of erection to achieve the purpose of rapid erection.

In the paper, it makes dynamics analysis and dynamics simulation research for the Leveraged Balance on Erecting mechanism by using ADAMS, and a better plan of electric cylinder movement rules is choosen by contrasting the forces on the key joints. The force of the key points is improved due to the trajectory planning that five curces of the movement rules will be selected.Then by comparing the experimental and simulation, the result proves the conclusion is correct .

\section{Mathematical Modeling for Erecting Mechanism}

The Leveraged Balance on Erecting mechanism has a short Electric Cylinders and on multistage cylinder change-stage impact on structure compared to direct Push structure. The structure is mainly consist of triangle arm $O_{2} A B$, Electric Cylinders $O_{1} A$ which endpoint $\mathrm{A}$ of the triangle arm is connecting to endpoint $O_{1}$ of the triangle arm, and connecting rod $\mathrm{BC}$ which make point $\mathrm{O}$ that the load rotate around as the fulcrum. Movement schematic of the structure is shown in figure 1. 


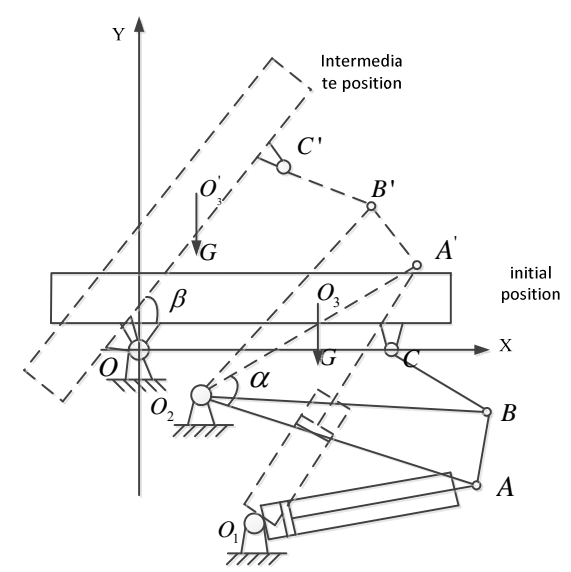

Fig. 1 The initial and intermediate position of erecting mechanism

Absolute coordinate system, which Coordinate origin is point $\mathrm{O}$, is built as is displayed in figure 1 . Assuming that the coordinates of point $\mathrm{I}$ is $\left(x_{I}, y_{I}\right)$, where $O_{3}$ represents the load centroid, $L_{J K}$ represents the length of JK, G represents the load weight of structure. When triangle arm $\mathrm{O}_{2} A B$ has rotated $\alpha$ to position $\mathrm{O}_{2} A^{\prime} B^{\prime}$, OC rotated $\beta$ to position $O C^{\prime}$. The whole process can be describe with the equation as follow:

$$
\left\{\begin{array}{l}
\left(\begin{array}{l}
x_{B^{\prime}}-x_{O_{2}} \\
y_{B^{\prime}}-y_{O_{2}}
\end{array}\right)=\left(\begin{array}{cc}
\cos \alpha & -\sin \alpha \\
\sin \alpha & \cos \alpha
\end{array}\right)\left(\begin{array}{l}
x_{B}-x_{O_{2}} \\
y_{B}-y_{O_{2}}
\end{array}\right) \\
\left(\begin{array}{l}
x_{C^{\prime}} \\
y_{C^{\prime}}
\end{array}\right)=\left(\begin{array}{cc}
\cos \beta & -\sin \beta \\
\sin \beta & \cos \beta
\end{array}\right)\left(\begin{array}{l}
x_{C} \\
y_{C}
\end{array}\right) \\
\sqrt{\left(x_{B^{\prime}}-x_{C^{\prime}}\right)^{2}+\left(y_{B^{\prime}}-y_{C^{\prime}}\right)^{2}} \\
=\sqrt{\left(x_{B}-x_{C}\right)^{2}+\left(y_{B}-y_{C}\right)^{2}}
\end{array}\right.
$$

It can be calculated from equations 1 that the relationship between the angle $\alpha$ and the coordinates of point $B$, point $C$ and the angle $\beta$ by eliminating the variables: $x_{B^{\prime}} y_{B^{\prime}} x_{C^{\prime}} y_{C^{\prime}}$.

Assumed that the length of the electric cylinder is as follow:

$M=L_{\mathrm{O}_{1} \mathrm{O}_{2}}^{2}+L_{\mathrm{O}_{2} \mathrm{~A}}^{2}-2 \cdot L_{\mathrm{O}_{1} \mathrm{O}_{2}} \cdot L_{\mathrm{O}_{2} \mathrm{~A}} \cdot \cos (\theta+\alpha)(2)$

Electric cylinder extending length is:

$$
s=\sqrt{M}-L_{O_{1} A}
$$

The velocity of electric cylinder extending:

$$
v=\dot{s}=\frac{L_{\mathrm{O}_{1} \mathrm{O}_{2}} \cdot L_{\mathrm{O}_{2} \mathrm{~A}} \cdot \sin (\theta+\alpha)}{\sqrt{M}} \dot{\alpha}
$$

The friction torques will be ignored in consideration of the mass of the linkage, the electric cylinder and triangle arm is small ${ }^{[8]}$. Taking the electric cylinder rotation and the triangle arm rotation as the object of study, the following equations will be get:

$$
\left\{\begin{array}{l}
J \cdot \ddot{\beta}=F_{B C}^{\prime} \cdot \sin \left(\angle O C^{\prime} B^{\prime}\right) \cdot L_{O C}-G \cdot \cos (\beta+\psi) \\
F_{C B}^{\prime} \cdot \sin \left(\angle O_{2} B^{\prime} C^{\prime}\right) \cdot L_{O_{2} B}=F_{O_{1} A}^{\prime} \cdot \sin \left(\angle O_{1} A^{\prime} O_{2}\right) \cdot L_{A O_{2}}
\end{array}\right.
$$

And the erecting force can be converted to:

$$
F=F_{O_{1} A}=\frac{J \cdot \ddot{\beta}+G \cdot \cos (\beta+\psi)}{\sin \left(\angle O C^{\prime} B^{\prime}\right) \cdot L_{O C}} \cdot \frac{\sin \left(\angle O_{2} B^{\prime} C^{\prime}\right) \cdot L_{O_{2} B}}{\sin \left(\angle O_{1} A^{\prime} O_{2}\right) \cdot L_{A O_{2}}}
$$

with 


$$
\left\{\begin{array}{l}
\angle O C^{\prime} B^{\prime}=\arccos \frac{L_{O C^{\prime}}^{2}+L_{B^{\prime} C^{\prime}}-\left(x_{B^{\prime}}^{2}+y_{B^{\prime}}^{2}\right)}{2 \cdot L_{O C^{\prime}} \cdot L_{B^{\prime} C^{\prime}}} \\
\angle O_{2} B^{\prime} C^{\prime}=\arccos \frac{L_{O_{2} B^{\prime}}^{2}+L_{B^{\prime} C^{\prime}}^{2}-\left(x_{C^{\prime}}-x_{O_{2}}\right)^{2}-\left(y_{C^{\prime}}-y_{O_{2}}\right)^{2}}{2 \cdot L_{O_{2} B^{\prime}} \cdot L_{B^{\prime} C^{\prime}}} \\
\angle O_{1} A^{\prime} O_{2}=\arcsin \left(\frac{L_{O_{1} O_{2}}}{\sqrt{M}} \sin (\alpha+\theta)\right)
\end{array}\right.
$$

We can calculate the relationship between the erecting force and Angular acceleration which the movement of the structure with the equation set $(1) \sim(7)$.

The simulation model on ADAMS of erecting mechanism ${ }^{[9]}$

Combined with practical and analytical, we can set up the simulation model that erecting mechanism on ADAMS virtual software ${ }^{[10]}$ is shown as in figure 2.

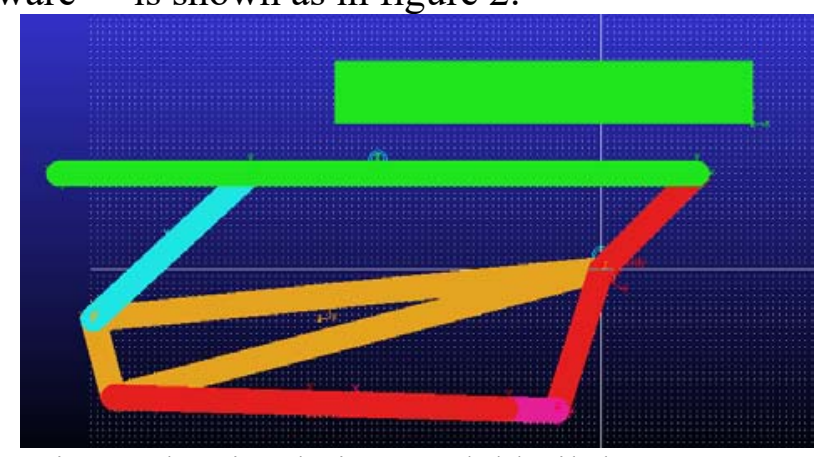

Fig. 2 The simulation model built by ADAMS

The position of all hinge points and the length of the linkage and the triangle arm side are shown as following:

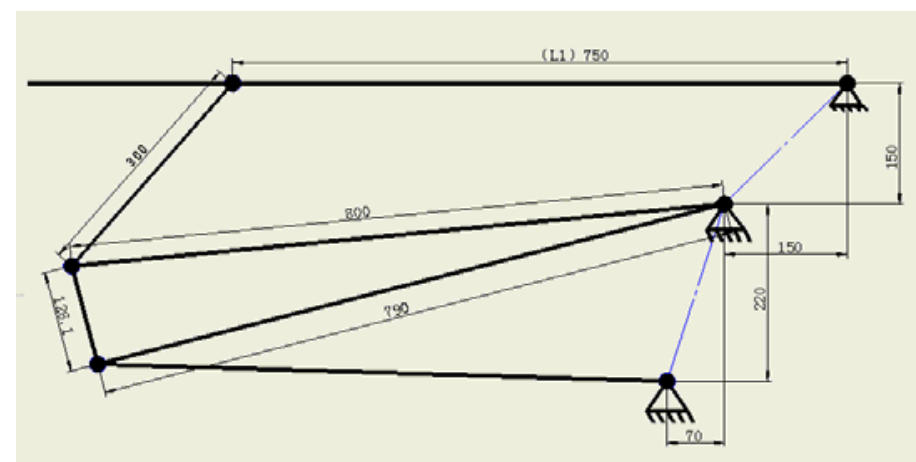

Fig. 3 Basic parameters of the erecting mechanism

To complete the simulation of erecting process, rotating motion which makes the electric cylinder rotate is added on the joint $O_{1}$ between the electric cylinder and the ground.

\section{The analysis of the different motion}

\section{The setting of relationship between erecting time and movement rules.}

In the paper, there will be five methods that the relationship between the erecting angle $\beta$ and the time $t$ is set by function ${ }^{[11]}$. Then the driving force can be measured by sensor and the loads of the structure on key joints will be obtained. The five methods are:

1. Uniform angular velocity motion.

2. Uniform angular acceleration -- uniform angular deceleration motion.

3. Uniform angular acceleration -- uniform angular velocity -- uniform angular deceleration motion.

4. Semi-cycle cosine acceleration motion.

5. The polynomial expression of quintics angular motion. 


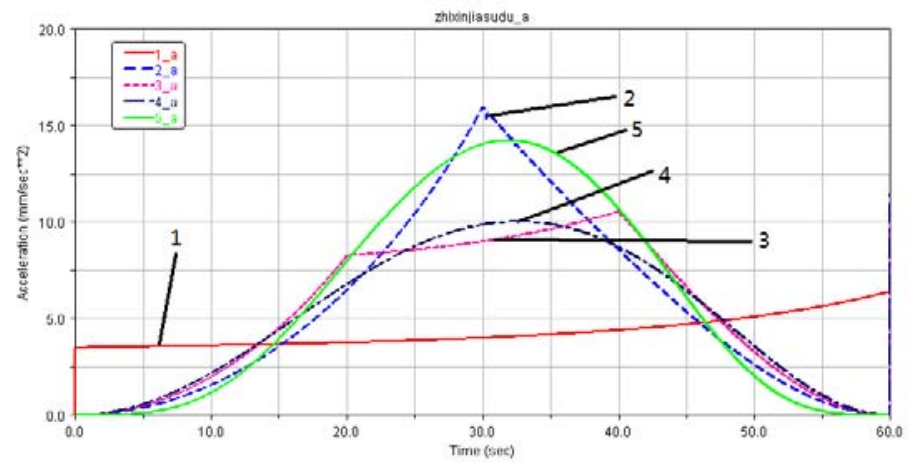

Fig. 4 The five curves of the acceleration

The control of motion in ADAMS. Rotating motion is added on the rotating joint $O_{3}$, and the method of proportional closed-loop control is adopted, where the reference angle is as input of the control system, and the difference between the reference angle and the actual angle $\beta$ as input of the scaling,then driving the erecting mechanism by enlarging it. So there will be have an impact on the angle $\beta$ which is as an output. The closed-loop control is structured as is shown in Fig. 5.

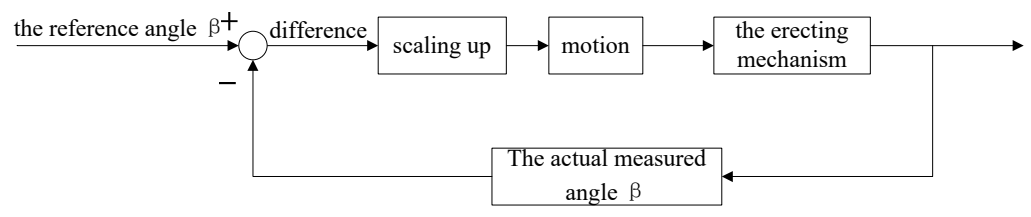

Fig. 5 block diagram of Proportional closed-loop control

Control Toolkit in ADAMS is used to complete the proportional closed-loop control system, in which the desired angle $\beta$ being set as one of the five movement rules mentioned above.
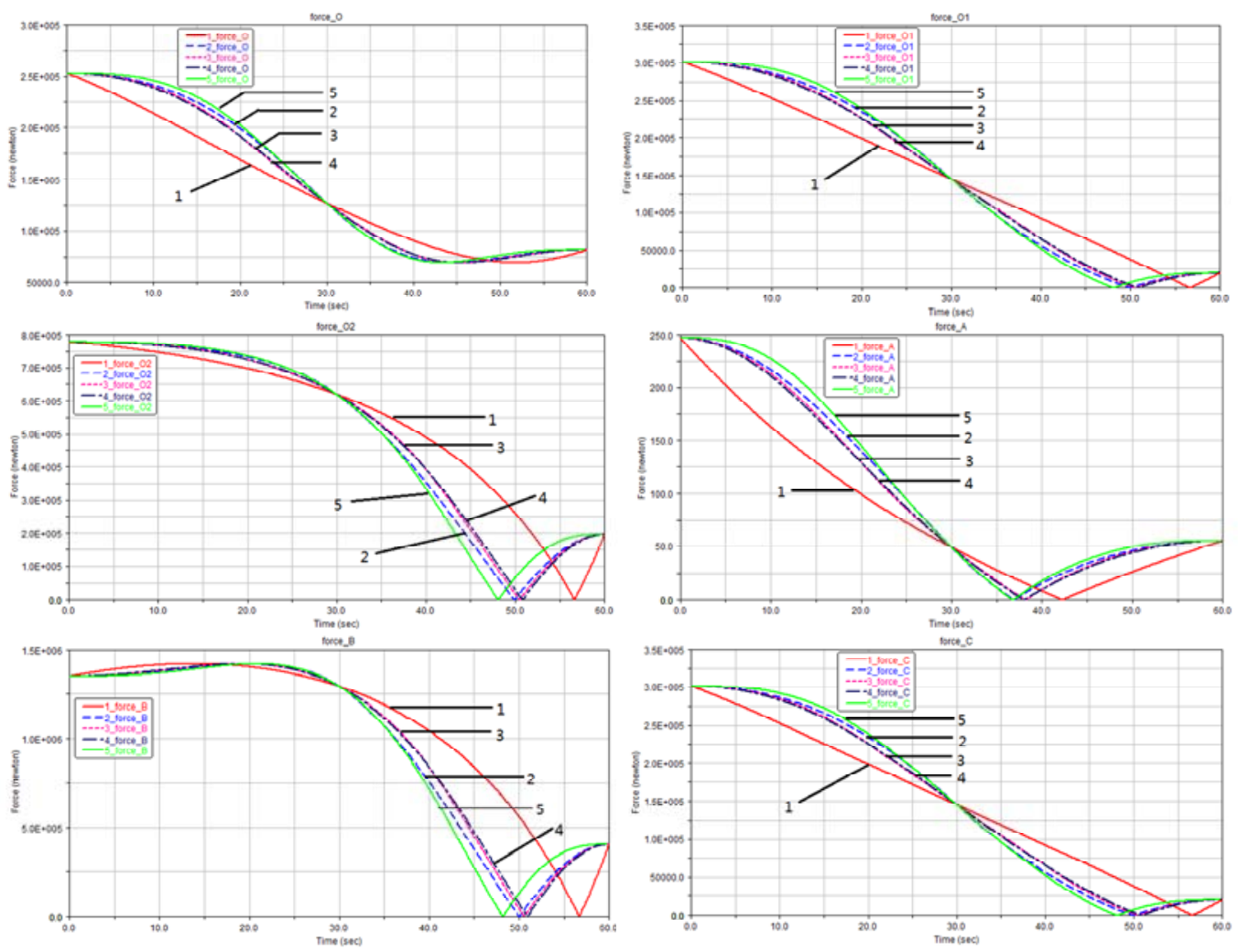

Fig. 6 The curves of the changing loads on key joints

The analysis of the simulation and experiment. Setting up the total time of the erecting progress $\mathrm{T}=60 \mathrm{~s}$, the curves of the changing loads on key joints $\mathrm{O} \sim \mathrm{C}$ and the acceleration of the center of the load gravity which is rotating are obtained after the dynamics simulation completed as is shown in Fig. 4. Curve 1 5 as is shown in Fig.6 represent the results under the trajectory planning of uniform angular velocity motion, uniform angular acceleration -- uniform angular deceleration motion, 
uniform angular acceleration -- uniform angular velocity -- uniform angular deceleration motion, semi-cycle cosine acceleration motion, quintic polynomial angular motion.

Combined with existing laboratory equipment, the platform is shown as Fig. 7 and the curves of the erecting force on simulation and experiments is shown as Fig.8.
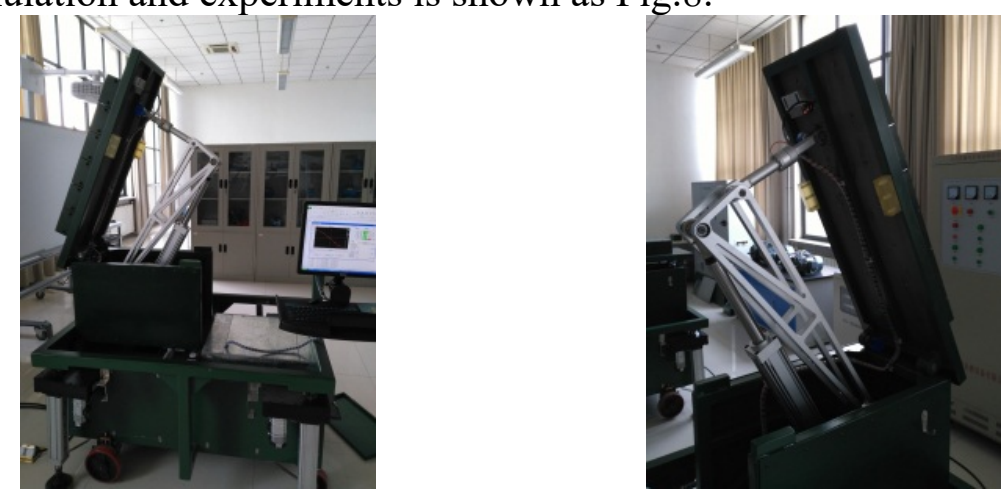

Fig.7 The experiment platform of the erecting mechanism

1. By contrast of simulation and experiment, we can get conclusion that the model is correct that the erecting force is from large to small, and then convert to negative.

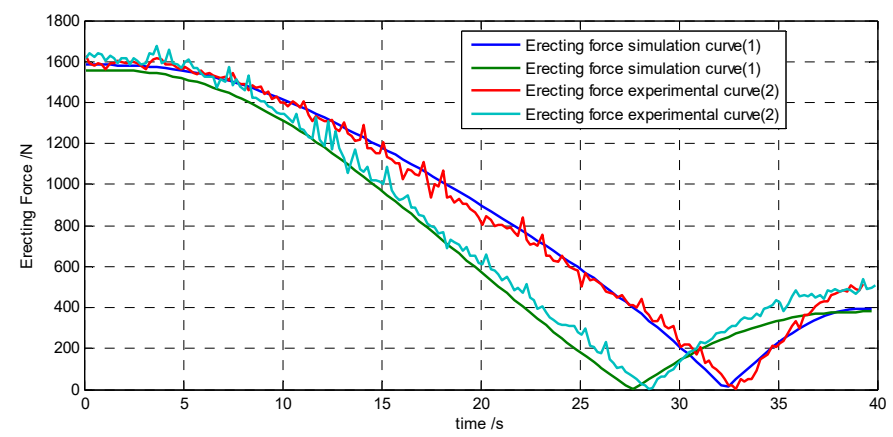

Fig. 8 The curves of the erecting force on simulation and experiments

2. The changing loads at the erecting process on six joints at almost the same force, and the difference of the maximum of loads at each time are little under the 5 different trajectory planning.

3. The curves of the angle acceleration of the load which is rotating can reflect the load's stress status, ranging them from large to small is: curve 2, curve 5, curve 3 , curve 4 , curve 1 .

\section{Summary}

Through the contrast analysis of different trajectory planning on the electric Leveraged Balance on Erecting mechanism, we can draw the conclusions that:

1. The simulation with ADAMS and experiments analysis provides reference to optimize the motion of erecting mechanism.

2. It reasonable to choose the semi-cycle cosine acceleration movement compared to the others. After the optimization, the changing loads on six joints are relatively small and the angle acceleration of the load center of gravity is smoothly running.

\section{References}

[1] W.H. Tang, S.M. Wang, and R. Yuan: Journal of Projectiles, Rockets, Missiles and Guidance, Vol. 27 (2007) No.2, p.325.

[2] Q.Q. Xia. Design for the structure and dynamic analysis of the kinetic energy missile launching device[D]. NANJING UNIVERSITY OF SCIENCE \& TECHNOLOGY, Vol. 01 (2015),p.8.

[3] G. Tian, J. Xie, and Y.N. Chen: Computer Simulation, Vol. 29 (2012) No.1, p.41.

[4] J.T. Feng, X.S. Guo, R.L. Shi, and B. Li: Machinery Design \& Manufacture, Vol. 6 (2013), p.193. 
[5] L. LI, F. LI, Y.B. Feng, et al. Research on Control Strategy of Large Hydraulic Erecting System Based on Adaptive Fuzzy Sliding Mode[J]. Acta Armamentarii, Vol. 37(2016),p.71.

[6] X.X. Zhu: Optimization Design For Dump Truck Lifting Mechanism Based On Virtual Prototype Technology (MS., Wuhan University of Technology, China 2010), p.48.

[7] X.Y. WU. Study on Electric Vertical Erection for Large Missile Launcher on Board[D]. Beijing: Beijing Institute of Techno- logy, Vol.6 (2015).

[8] M.B. LI, X.Q. Chang, J. Liu. Research on Power Matching Performance of Missile Vertical Erecting Mechanism[J]. Tactical Missile Technoligy, Vol.3(2012),p.27.

[9] C.Z. Liu: The Research and Simulation of the Medium Erection Device (MS., Huazhong University of Science and Technology, China 2007), p.15.

[10] X.L. Yao: A Study of the Dumping Stability of the Dump Tractor-semitrailer Based on Multi-body Dynamic (MS., Wuhan University of Technology, China 2008), p.33.

[11] P. Feng. Introduction for the structure and dynamic research of the vehicle missile launching device[D]. NANJING UNIVERSITY OF SCIENCE \& TECHNOLOGY, Vol. 1(2015). 